\title{
Minor-axis velocity gradients in spirals and the case of inner polar disks ${ }^{\star} \star \star \star$
}

\author{
E. M. Corsini, A. Pizzella, L. Coccato, and F. Bertola \\ Dipartimento di Astronomia, Università di Padova, vicolo dell’Osservatorio 2, 35122 Padova, Italy \\ Received 4 March 2003 / Accepted 3 June 2003
}

\begin{abstract}
We measured the ionized-gas and stellar kinematics along the major and minor axis of a sample of 10 early-type spirals. Much to our surprise we found a remarkable gas velocity gradient along the minor axis of 8 of them. According to the kinematic features observed in their ionized-gas velocity fields, we divide our sample galaxies in three classes of objects. (i) NGC 4984, NGC 7213, and NGC 7377 show an overall velocity curve along the minor axis without zero-velocity points, out to the last measured radius, which is interpreted as due to the warped structure of the gaseous disk. (ii) NGC 3885, NGC 4224 , and NGC 4586 are characterized by a velocity gradient along both major and minor axis, although non-zero velocities along the minor axis are confined to the central regions. Such gas kinematics have been explained as being due to non-circular motions induced by a triaxial potential. (iii) NGC 2855 and NGC 7049 show a change of slope of the velocity gradient measured along the major axis (which is shallower in the center and steeper away from the nucleus), as well as non-zero gas velocities in the central regions of the minor axis. This has been attributed to the presence of a kinematically-decoupled gaseous component in orthogonal rotation with respect to the galaxy disk, namely an inner polar disk. The case and origin of inner polar disks are discussed and the list of their host galaxies is presented.
\end{abstract}

Key words. galaxies: kinematics and dynamics - galaxies: spiral - galaxies: structure

\section{Introduction}

As a result of the analysis of the kinematical data available in the literature for the ionized-gas component of the S0s and spirals listed in the Revised Shapley-Ames Catalog of Bright Galaxies (Sandage \& Tammann 1981, hereafter RSA), we realized that $\sim 60 \%$ of unbarred galaxies show a remarkable gas velocity gradient along their optical minor axis. This phenomenon is observed all along the Hubble sequence of disk galaxies, but it is particularly frequent in early-type spirals (Coccato et al. 2003). However, such a minor-axis velocity gradient is unexpected if gas is moving onto circular orbits in a disk coplanar to the stellar one.

In a non-axisymmetric potential, gas in equilibrium moves onto closed elliptical orbits which become nearly circular at larger radii (de Zeeuw \& Franx 1989; Gerhard et al. 1989). A velocity gradient along the apparent minor axis of the galaxy is observed if the inner gas orbits are seen at intermediate angle between their intrinsic major and minor axes. This is the case of bulges, since their intrinsic shape is generally triaxial both

\footnotetext{
Send offprint requests to: E. M. Corsini, e-mail: corsini@pd.astro.it

* Based on observations carried out at the European Southern Observatory (ESO 62.A-0463 and 63.N-0305).

$\star \star$ Tables 3 and 4 are only available in electronic form at the CDS via anonymous ftp to cdsarc.u-strasbg.fr $(130.79 .128 .5)$ or via http://cdsweb.u-strasbg.fr/cgi-bin/qcat?J/A+A/408/873
}

in barred (Kormendy 1982) and in unbarred galaxies (Bertola et al. 1991). Detailed velocity fields for the gaseous component have been obtained and modeled to demonstrate bulge triaxiality only for our Galaxy (Gerhard \& Vietri 1986), and two external galaxies, namely NGC 4845 (Bertola et al. 1989; Gerhard et al. 1989) and M31 (Berman 2001 and references therein).

A gas velocity gradient along the apparent minor axis of the galaxy is observed if the gaseous component is not settled onto the galaxy disk, as in the case of kinematically-decoupled gaseous components and warped gaseous disks. The presence of a kinematically-decoupled component is usually interpreted as the relic of accretion events that occurred during the host galaxy lifetime. The nature and the orientation of the decoupled component mostly depend on the initial angular momentum and mass of the accreted material. Polar rings (e.g. Whitmore et al. 1990), counterrotating disks (see Galletta 1996; Bertola \& Corsini 1999 for a review) and orthogonally-rotating cores (e.g. Bertola \& Corsini 2000; Sil'chenko 2003) are examples of kinematically-decoupled structures observed in disk galaxies, most of which display an otherwise undisturbed morphology.

A misalignment between the angular momenta of inner and outer parts characterizes a warped disk. This phenomenon has been observed in at least half of all the spiral galaxies and has been explained both with an internal and external origin (see Binney 1992 for a review). Significant warps are usually measured in the cold gaseous component, while they are less frequent in the stellar one. Gaseous and stellar disks are generally 
Table 1. Parameters of the sample galaxies.

\begin{tabular}{|c|c|c|c|c|c|c|c|c|c|}
\hline Name & \multicolumn{2}{|c|}{ Morphological type } & & $V_{\odot}$ & & $M_{B_{\mathrm{T}}}^{0}$ & $D_{25} \times d_{25}$ & $i$ & Minor-axis region \\
\hline (1) & $\begin{array}{c}\text { [RSA] } \\
(2)\end{array}$ & $\begin{array}{c}{[\mathrm{RC} 3]} \\
(3)\end{array}$ & $\begin{array}{l}{\left[{ }^{\circ}\right]} \\
(4)\end{array}$ & $\begin{array}{c}{\left[\mathrm{km} \mathrm{s}^{-1}\right]} \\
(5)\end{array}$ & $\begin{array}{c}{[\mathrm{Mpc}]} \\
(6)\end{array}$ & $\begin{array}{c}{[\mathrm{mag}]} \\
(7)\end{array}$ & (8) & $\begin{array}{l}{\left[{ }^{\circ}\right]} \\
(9)\end{array}$ & $\begin{array}{c}\text { where } V_{\mathrm{g}} \neq 0 \\
(10)\end{array}$ \\
\hline NGC 1638 & $\mathrm{Sa}$ & $\mathrm{SAB} 0(\mathrm{rs}) ?$ & 70 & $3258 \pm 11$ & 43.5 & -20.42 & $2.0 \times 1{ }^{\prime} .5$ & 43 & $?$ \\
\hline NGC 2855 & $\mathrm{Sa}(\mathrm{r})$ & (R)S0/a(rs) & 120 & $1897 \pm 17$ & 22.3 & -19.45 & $2.5 \times 2 ! .2$ & 27 & inner \\
\hline NGC 3885 & $\mathrm{Sa}$ & $\mathrm{S} 0 / \mathrm{a}(\mathrm{s})$ & 123 & $1952 \pm 8$ & 22.3 & -19.44 & $2{ }^{\prime} 4 \times 1^{\prime} .0$ & 69 & inner \\
\hline NGC 4224 & $\mathrm{Sa}$ & $\mathrm{Sa}(\mathrm{s}): \mathrm{sp}$ & 57 & $2624 \pm 6$ & 33.0 & -20.07 & $2^{\prime} .6 \times 1{ }^{\prime} .0$ & 69 & inner \\
\hline NGC 4235 & $\mathrm{Sa}$ & $\mathrm{Sa}(\mathrm{s}) \mathrm{sp}$ & 48 & $2249 \pm 11$ & 28.1 & -20.35 & $4^{\prime} .2 \times 0.9$ & 82 & $?$ \\
\hline NGC 4586 & $\mathrm{Sa}$ & $\mathrm{Sa}(\mathrm{s}): \mathrm{sp}$ & 115 & $815 \pm 7$ & 17.0 & -19.04 & $4.0 \times 1.3$ & 73 & inner \\
\hline NGC 4984 & $\mathrm{Sa}(\mathrm{s})$ & (R)SAB0 $0^{+}$ & 90 & $1279 \pm 6$ & 14.2 & -18.73 & $2^{\prime} .8 \times 2^{\prime} .2$ & 38 & extended \\
\hline NGC 7049 & $\mathrm{SO}_{3}(4) / \mathrm{Sa}$ & $\mathrm{SO}(\mathrm{s})$ & 57 & $2285 \pm 6$ & 29.6 & -20.79 & $4^{\prime} .3 \times 3^{\prime} .0$ & 47 & inner \\
\hline NGC 7213 & $\mathrm{Sa}(\mathrm{rs})$ & $\mathrm{Sa}(\mathrm{s}):$ & 124 & $1784 \pm 6$ & 23.1 & -20.69 & $3: 1 \times 2{ }^{\prime} .8$ & 27 & extended \\
\hline NGC 7377 & $\mathrm{SO}_{2 / 3} / \mathrm{Sa} \mathrm{pec}$ & $\mathrm{S}^{+}(\mathrm{s})$ & 101 & $3339 \pm 11$ & 45.7 & -21.37 & $3{ }^{\prime} 0 \times 22^{\prime} .5$ & 34 & extended \\
\hline
\end{tabular}

Notes. - Column 2: morphological classification from RSA. Column 3: morphological classification from RC3. Column 4: major-axis position angle from RC3. Column 5: heliocentric systemic velocity derived from the center of symmetry of the rotation curve of the gas along the galaxy major axis. Column 6: distance obtained as $V_{0} / H_{0}$ with $H_{0}=75 \mathrm{~km} \mathrm{~s}^{-1} \mathrm{Mpc}^{-1}$ and $V_{0}$ the systemic velocity derived from $V_{\odot}$ corrected for the motion of the Sun with respect of the Local Group according to the RSA. For NGC 4586 we assumed the distance of the Virgo Cluster by Freedman et al. (1994). Column 7: absolute total blue magnitude corrected for inclination and extinction from RC3. Column 8: major and minor isophotal diameters from RC3. Column 9: inclination derived from $\cos ^{2} i=\left(q^{2}-q_{0}^{2}\right) /\left(1-q_{0}^{2}\right)$ assuming the observed axial ratio $q=d_{25} / D_{25}$ from RC3 and the typical intrinsic axial ratio for S0/Sa and Sa galaxies $\left(q_{0}=0.18\right.$, Guthrie 1992). Column 10: minor-axis region where non-zero velocity is measured for the ionized gas. Inner = non-zero velocity is confined to the nuclear region; extended $=$ non-zero velocity is observed out to the last measured radius; ? = doubtful velocity gradient.

coplanar and flat in the optical region and their warp is observed at larger radii (Briggs 1990; Bosma 1991), though noncoplanar gas and stellar disks have been seen even in the optical region of some galaxies giving rise to a minor-axis velocity gradient. For example, in a triaxial potential bulge (or in a bar) which is tumbling about the short axis, the gas leaves the plane perpendicular to the rotation axis and settles onto the so-called anomalous orbits (van Albada et al. 1982). These closed orbits are fixed in the figure even though the figure rotates. They are nearly planar and fairly circular, but their inclination with respect to the figure rotation axis increases with the orbital radius. This explains the tilted disks of counterrotating gas observed in some SB0 galaxies (e.g. Emsellem \& Arsenault 1996).

This paper aims to investigate the presence of non-circular and off-plane gas motions in bulges. These phenomena have to be taken into account as well as the presence of pressuresupported ionized gas (Bertola et al. 1995; Cinzano et al. 1999; Pignatelli et al. 2001) when the observed rotation velocity of the gas is adopted to trace the circular speed in mass modeling, in order to derive the distribution of luminous and dark matter in the innermost regions of disk galaxies.

To maximize the chance of detecting a gas velocity gradient along the minor axis we selected a sample of early-type spirals according to the results of Coccato et al. (2003). We randomly chose 10 galaxies among the Sa spirals listed in The Carnegie Atlas of Galaxies (Sandage \& Bedke 1994, hereafter CAG). A compilation from the literature of the properties of the sample galaxies is presented in Table 1. The images of the sample galaxies taken from the Digitized Sky Survey are given in Fig. 1. In a previous paper (Corsini et al. 2002) we reported on the special case of NGC 2855, which is characterized by the presence of a kinematically-decoupled component of ionized gas in orthogonal rotation with respect to the stellar disk as well as of a faint ring-like structure surrounding the galaxy. These features are indicative of an on-going acquisition process. Here we present results for the remaining 9 sample galaxies. The long-slit spectroscopic observations and data analysis are described in Sect. 2. The resulting stellar and ionized-gas kinematics are given in Sect. 3 and interpreted in Sect. 4. The case of inner polar disks is discussed in Sect. 4 too.

\section{Spectroscopic observations and data reduction}

\subsection{Spectroscopic observations}

The spectroscopic observations were carried out at the European Southern Observatory in La Silla (Chile) with the 1.52-m ESO telescope on March 17-22 (hereafter run 1) and September 09-12, 1999 (hereafter run 2). The telescope was equipped with the Boller \& Chivens Spectrograph. The No. 33 grating with 1200 grooves $\mathrm{mm}^{-1}$ was used in the first order in combination with a 2 !' $2 \times 4.2$ slit, and the No. 39 Loral/Lesser CCD with $2048 \times 2048$ pixels of $15 \times 15 \mu \mathrm{m}^{2}$. The spectral range between about $4850 \AA$ and $6850 \AA$ was covered with a reciprocal dispersion of $0.98 \AA$ pixel $^{-1}$. The spatial scale was $0^{\prime} 81 \mathrm{pixel}^{-1}$. The instrumental resolution was $2.75 \AA$ (FWHM) and it was measured from the mean FWHM of arc lines in wavelength-calibrated comparison spectra. It corresponds to $\sigma_{\text {instr }} \approx 50 \mathrm{~km} \mathrm{~s}^{-1}$ at $\mathrm{H} \alpha$. Repeated exposures (typically of $3600 \mathrm{~s}$ each) were taken along both major and minor axes of all the sample galaxies, after centering their nucleus on the slit using the guiding TV camera. The observed position angles and corresponding exposure times are given in Table 2. In each observing run we obtained spectra of late- $\mathrm{G}$ to early-K giant stars to be used as templates in measuring the stellar 

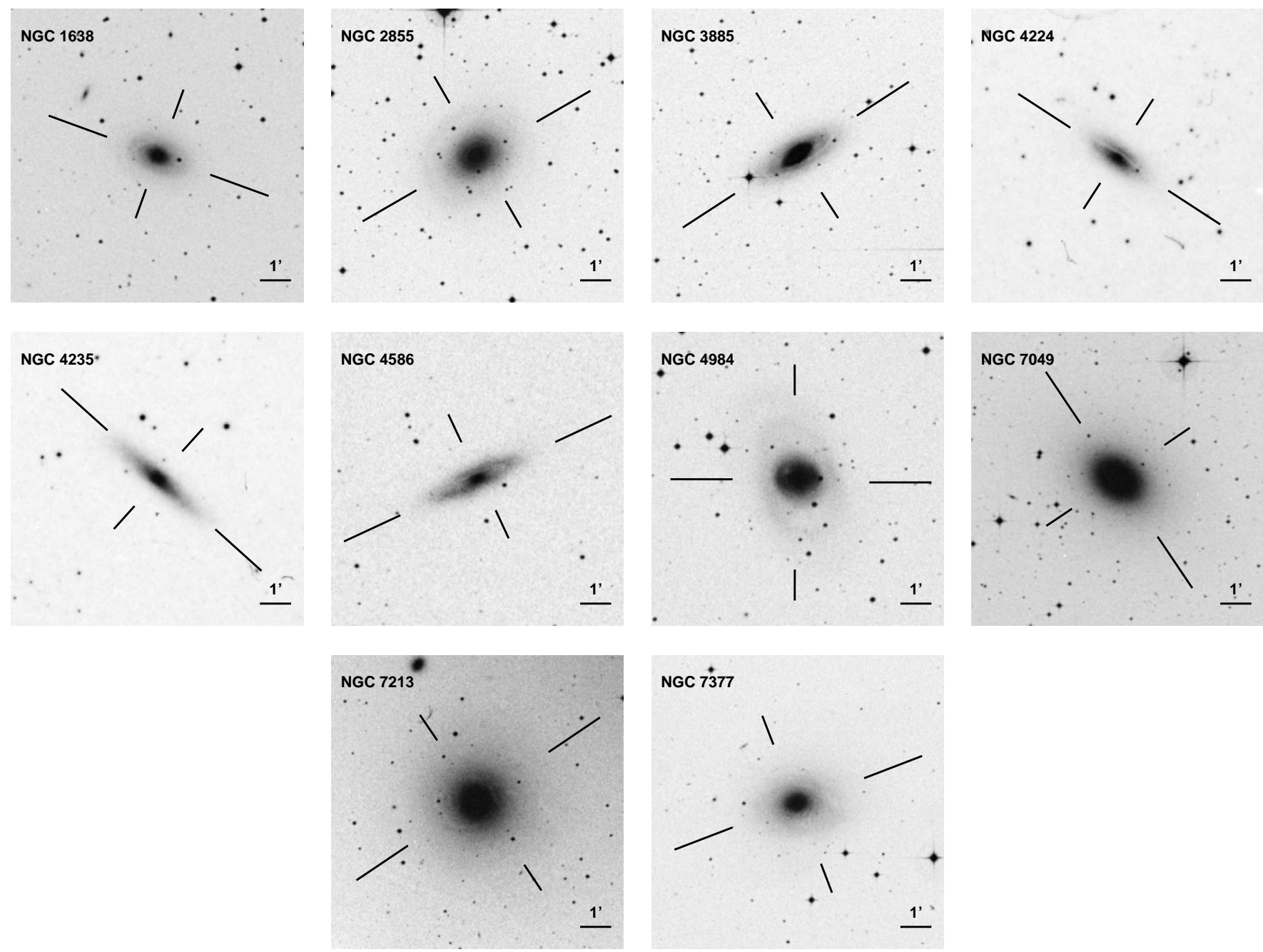

Fig. 1. Optical images of the sample galaxies taken from the Digitized Sky Survey. The orientation of the images is north up and east left and each image is $8^{\prime} \times 8^{\prime}$. Long and short lines mark the position of optical major and minor axis according to RC 3 catalog.

kinematics. A spectrum of the comparison helium-argon lamp was taken before and/or after every object exposure to ensure an accurate wavelength calibration. Seeing FWHM during the observing nights ranged between $1^{\prime \prime}$ and $3^{\prime \prime}$ as measured by the ESO Differential Image Motion Monitor.

\subsection{Data reduction}

Basic data reduction was performed as in Corsini et al. (1999). Using standard ESO-MIDAS ${ }^{1}$ routines, all the spectra were bias subtracted, flat-field corrected by quartz lamp and twilight exposures, cleaned of cosmic rays, and wavelength calibrated. After calibration, the different spectra obtained for a given galaxy along the same position angle were co-added using the center of the stellar-continuum radial profile as a reference. The contribution of the sky was determined from the outermost $\sim 10^{\prime \prime}$ at the two edges of the resulting frames where the galaxy light was negligible, and then subtracted.

${ }^{1}$ MIDAS is developed and maintained by the European Southern Observatory.
The ionized-gas kinematics were measured from the [N II] $\lambda \lambda 6548,6583, \mathrm{H} \alpha$ and [S II] $\lambda \lambda 6716,6731$ emission lines by means of the MIDAS package ALICE as done by Corsini et al. (1999). The position and FWHM of each emission line were determined by interactively fitting one Gaussian to each line plus a polynomial to its local continuum. The center wavelength of the fitting Gaussian was converted into velocity in the optical convention, then the standard heliocentric correction was applied. The Gaussian FWHM was corrected for the instrumental FWHM, and then converted into the velocity dispersion. In the regions where the intensity of the emission lines was low, we binned adjacent spectral rows in order to improve the signal-to-noise ratio, $\mathrm{S} / \mathrm{N}$, of the lines. We expressed the velocity and velocity dispersion errors as a function of the relevant line S/N ratio as done in Corsini et al. (1999) and derived ionized-gas velocity $\left(v_{\mathrm{g}}\right)$ and velocity dispersion $\left(\sigma_{\mathrm{g}}\right)$ as the weighted mean of values measured for the different emission lines. The kinematics of the ionized gas are reported in Table 3 and plotted in Fig. 2 for all the sample galaxies.

The stellar kinematics were obtained from the absorption features present in the wavelength range running from about $4960 \AA$ to $5540 \AA$ and centered on the Mg line triplet 
Table 2. Log of spectroscopic observations.

\begin{tabular}{|c|c|c|c|c|c|c|}
\hline Object & (2) & $\begin{array}{l}\text { PA } \\
{[0]} \\
(3)\end{array}$ & Position & $\begin{array}{l}\text { Single exp. time } \\
\qquad \begin{array}{c}{[\mathrm{s}]} \\
(5)\end{array}\end{array}$ & $\begin{array}{l}\text { Total exp. time } \\
\qquad \begin{array}{c}{[\mathrm{h}]} \\
(6)\end{array}\end{array}$ & Template \\
\hline \multirow[t]{2}{*}{ NGC 1638} & 2 & 70 & MJ & $3600+3000$ & 1.8 & HR 1318 \\
\hline & 2 & 160 & $\mathrm{MN}$ & $2 \times 2700$ & 1.5 & HR 1318 \\
\hline \multirow[t]{2}{*}{ NGC 2855} & 1 & 120 & MJ & $2 \times 3600$ & 2.0 & HR 2035 \\
\hline & 1 & 30 & $\mathrm{MN}$ & $3 \times 3600$ & 3.0 & HR 2035 \\
\hline \multirow[t]{2}{*}{ NGC 3885} & 1 & 123 & MJ & $4 \times 3600$ & 4.0 & HR 2429 \\
\hline & 1 & 33 & $\mathrm{MN}$ & $3 \times 3600$ & 3.0 & HR 2429 \\
\hline \multirow[t]{2}{*}{ NGC 4224} & 1 & 57 & MJ & $2 \times 3600$ & 2.0 & HR 2429 \\
\hline & 1 & 147 & $\mathrm{MN}$ & $2 \times 3600$ & 2.0 & HR 2429 \\
\hline \multirow[t]{2}{*}{ NGC 4235} & 1 & 48 & $\mathrm{MN}$ & $2 \times 3600$ & 2.0 & HR 2035 \\
\hline & 1 & 138 & $\mathrm{MN}$ & $2 \times 3600$ & 2.0 & HR 2035 \\
\hline \multirow[t]{2}{*}{ NGC 4586} & 1 & 115 & MJ & $4 \times 3600$ & 4.0 & HR 2701 \\
\hline & 1 & 25 & MN & $3 \times 3600$ & 3.0 & HR 2701 \\
\hline \multirow[t]{2}{*}{ NGC 4984} & 1 & 90 & MJ & $3 \times 3600+3000$ & 3.8 & HR 2035 \\
\hline & 1 & 0 & $\mathrm{MN}$ & $3 \times 3600$ & 3.0 & HR 2035 \\
\hline \multirow[t]{2}{*}{ NGC 7049} & 2 & 57 & MJ & 3600 & 1.0 & HR 2113 \\
\hline & 2 & 147 & $\mathrm{MN}$ & 3600 & 1.0 & HR 2113 \\
\hline \multirow[t]{2}{*}{ NGC 7213} & 2 & 124 & MJ & $2 \times 3600+2700$ & 2.8 & HR 2113 \\
\hline & 2 & 34 & $\mathrm{MN}$ & $2 \times 3600$ & 2.0 & HR 2113 \\
\hline \multirow[t]{2}{*}{ NGC 7377} & 2 & 101 & MJ & $2 \times 3600$ & 2.0 & HR 2113 \\
\hline & 2 & 21 & $\mathrm{MN}$ & $2 \times 3600$ & 2.0 & HR 2113 \\
\hline
\end{tabular}

Notes. - Column 2: observing run. Column 3: slit position angle measured North through East. Column 4: slit position. MJ = major axis; $\mathrm{MN}=$ minor axis. Column 5: number and exposure time of the single exposures. Column 6: total exposure time. Column 7: giant star used as kinematical template in measuring the stellar kinematics (see Sect. 2.2).

$(\lambda \lambda 5164,5173,5184 \AA)$. We used the Fourier Correlation Quotient method (FCQ, Bender 1990) following the prescriptions of Benderet al. (1994). The spectra were rebinned along the spatial direction to obtain a nearly constant signal-to-noise ratio larger than 20 per resolution element. The galaxy continuum was removed row-by-row by fitting a fourth to sixth order polynomial as in Bender et al. (1994). The stars adopted as kinematical templates are given in Table 2. This allowed us to derive, for each spectrum, the line-of-sight stellar velocity $\left(v_{\star}\right)$ and velocity dispersion $\left(\sigma_{\star}\right)$ by fitting a Gaussian to the line-of-sight velocity distribution (LOSVD) at each radius. Velocities were corrected for heliocentric velocity. We derived errors on the stellar kinematics from photon statistics and CCD read-out noise, calibrating them by Monte Carlo simulations as done by Gerhard et al. (1998). These errors do not take into account possible systematic effects due to template mismatch. The kinematics of the stellar component are reported in Table 4 and plotted in Fig. 2 for all the sample galaxies.

\section{The stellar and ionized gas kinematics}

The velocity curves and velocity dispersion profiles we measured for the gaseous and stellar components along the major and minor axis of the sample galaxies are presented in Fig. 2. The rotation velocities of stars $V_{\star}\left(\equiv\left|v_{\star}-V_{\odot}\right|\right)$ and ionized gas $V_{\mathrm{g}}\left(\equiv\left|v_{\mathrm{g}}-V_{\odot}\right|\right)$ given in Tables 3 and 4 and plotted in Fig. 2 are the observed velocities after subtracting the systemic velocities of Table 1 and without applying any correction for galaxy inclination. $\sigma_{\star}$ and $\sigma_{\mathrm{g}}$ are the velocity dispersion of stars and gas, respectively. The case of NGC 2855 is shown in Fig. 2 for the sake of completeness. For details the reader is referred to Corsini et al. (2002).

\subsection{Major-axis kinematics}

The only noteworthy features observed in the major-axis kinematics of the ionized-gas and stellar component are the counterrotation of gas with respect to stars found in NGC 7213, and the reversal of gas rotation measured in the outermost regions of NGC 7377. In the sample galaxies $V_{\star}$ has always a shallower gradient than $V_{\mathrm{g}}$ (except for the inner regions of NGC 2855 and NGC 7049, where $V_{\star}>V_{\mathrm{g}}$ ), and $V_{\star} \approx V_{\mathrm{g}}$ at the last observed radius (except for NGC 7213 and NGC 7377). Typically $\sigma_{\star}$ exceeds $150 \mathrm{~km} \mathrm{~s}^{-1}$ and it is larger than $\sigma_{\mathrm{g}}$ at all radii (except in the center of NGC 4224 and NGC 4235 where $\sigma_{\star} \lesssim \sigma_{\mathrm{g}}$ ). If we took into account only the major-axis data, we could straightforwardly explain the observed kinematical properties by a dynamical model where gas is confined in the disk and supported by rotation and stars mostly belong to the bulge and are supported by dynamical pressure.

\subsection{Minor-axis kinematics}

This is not the case if we consider the minor-axis kinematics too. The minor-axis velocity gradient we measure for the gaseous component of 8 out of 10 sample galaxies is hardly 

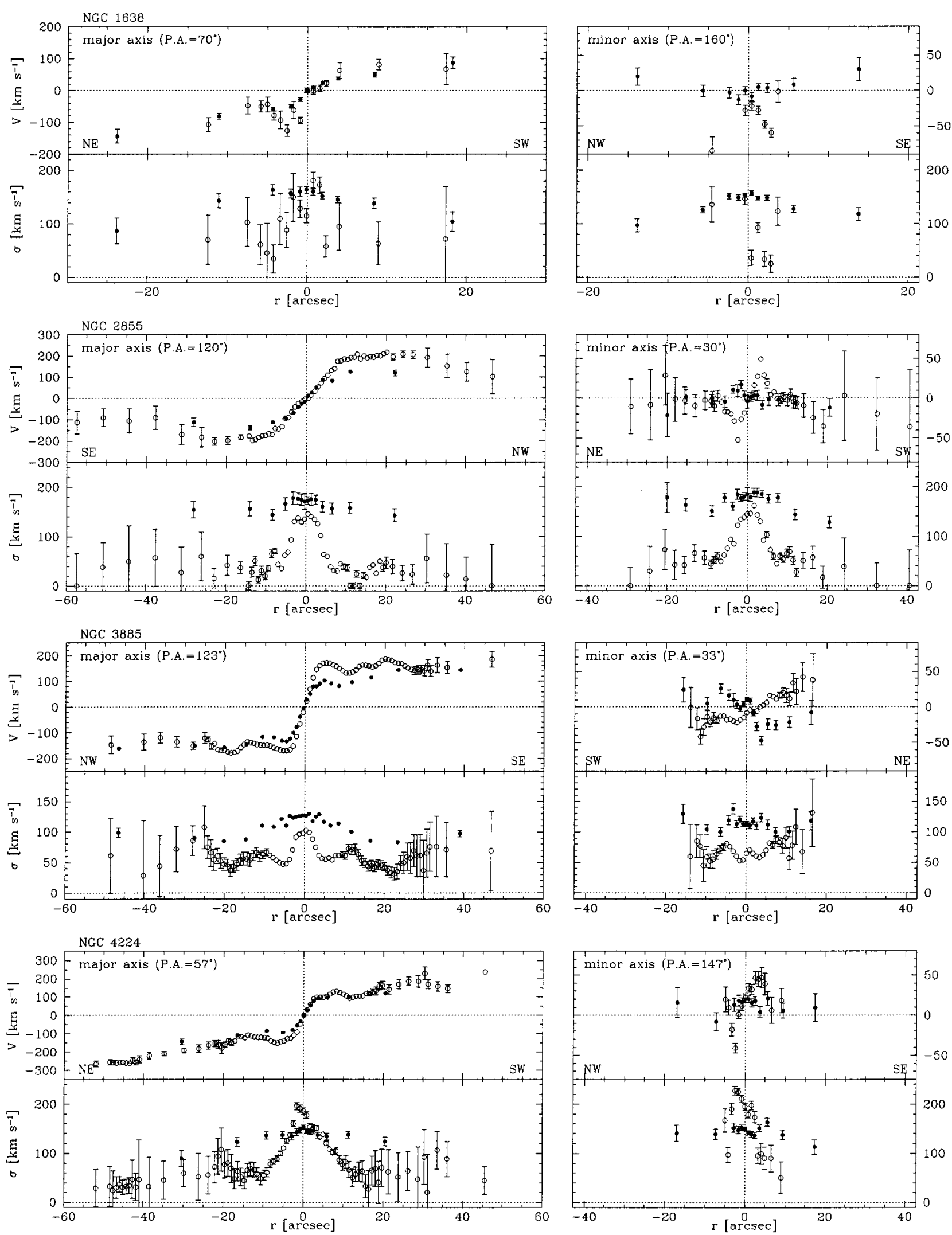

Fig. 2. Stellar (filled circles) and ionized-gas (open circles) kinematics measured along major (left panels) and minor (right panels) axis of the sample galaxies. Errorbars smaller than symbols are not plotted.

explained if we naively assume that the ionized gas is moving onto circular orbits in the galaxy disk.
Non-zero gas velocities are measured in the nuclear regions along the minor axis of NGC 2855, NGC 3885, NGC 4224, 

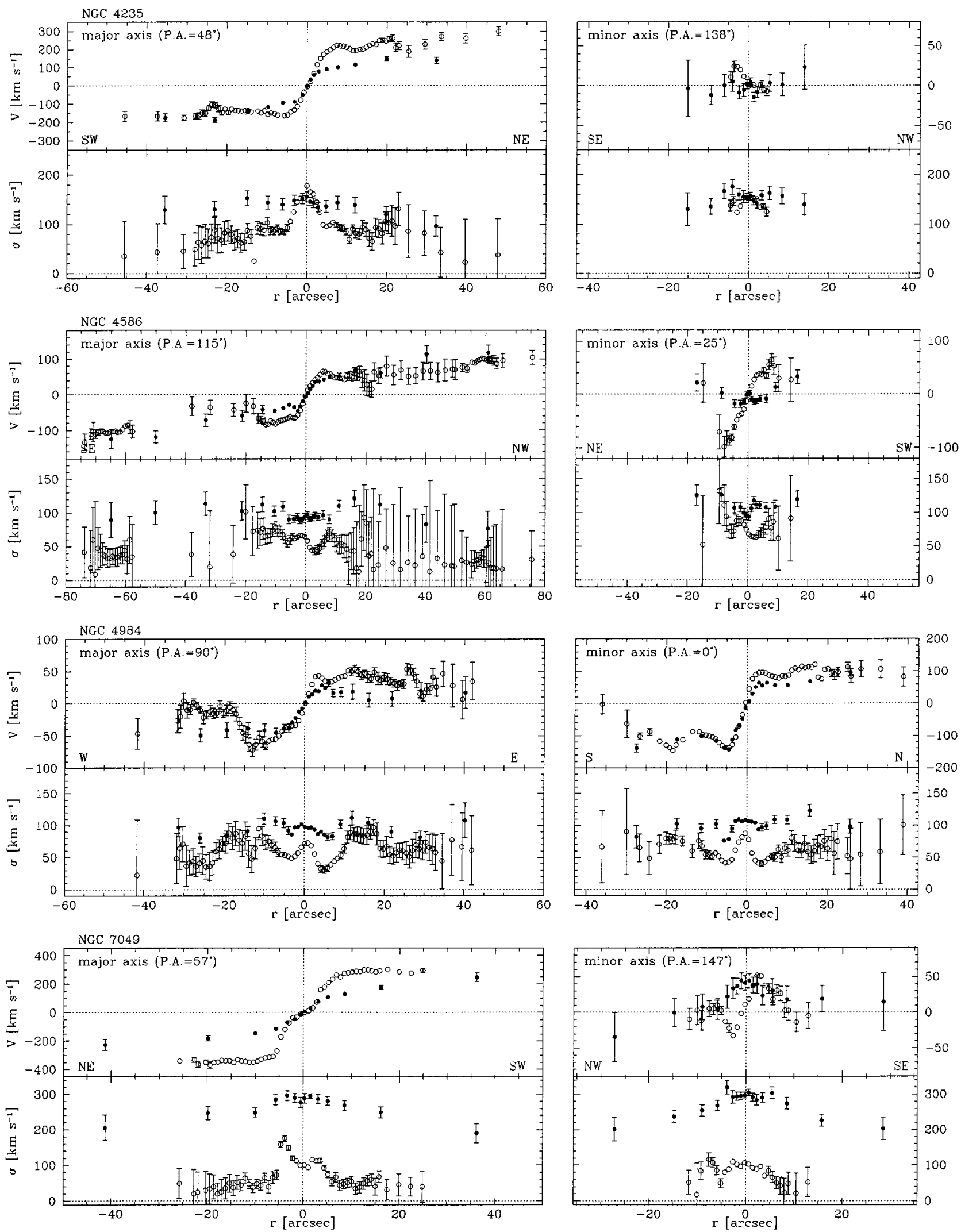

Fig. 2. continued.

NGC 4586, and NGC 7049. The gas velocity curve shows a steep gradient rising to a maximum observed velocity of $\approx 50 \mathrm{~km} \mathrm{~s}^{-1}$ in the inner few arcsec, then the velocity drops to $\approx 0 \mathrm{~km} \mathrm{~s}^{-1}$ at larger radii. Non-zero gas velocities are measured along the minor axis of NGC 4984, NGC 7213, and NGC 7377 all over the observed radial range. The maximum 

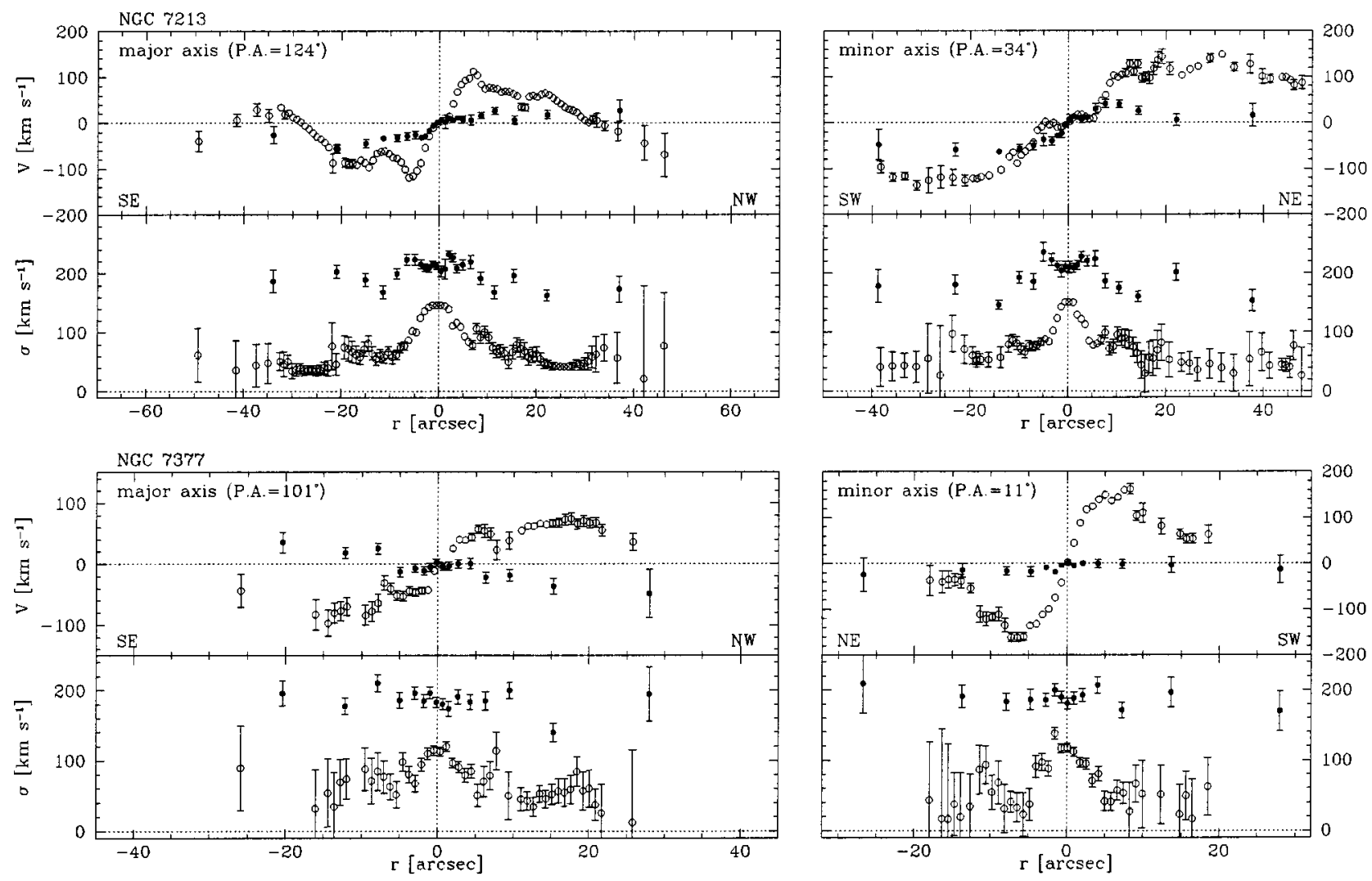

Fig. 2. continued.

velocity is $\gtrsim 150 \mathrm{~km} \mathrm{~s}^{-1}$ and it is even larger than the maximum value measured along the major axis. Finally, along the minor axis of the remaining 2 sample galaxies, namely NGC 1638 and NGC 4235, the gas is either too poorly detected or it shows a velocity curve that is too asymmetric to derive any conclusion. We will not consider these 2 galaxies further.

As far as the minor-axis stellar kinematics is concerned, no significant rotation velocity is observed along the minor axes of NGC 1638, NGC 2885, NGC 4235, and NGC 7377. The same is true for NGC 4224 and NGC 7049 if we interpret the falling of the stellar velocity from a central maximum of $\approx 30 \mathrm{~km} \mathrm{~s}^{-1}$ to $0 \mathrm{~km} \mathrm{~s}^{-1}$ off the center as due to an offset position of the slit of $\approx 1^{\prime \prime}$ with respect to the nucleus and parallel to the minor axis. Non-zero stellar velocities are measured along the minor axis of NGC 4984 and NGC 7213 over all the observed radial range. For these galaxies the amplitude of the stellar velocity curve measured along the minor axis is larger than that measured along the major axis. Stars are counterrotating with respect to gas along the minor axis of NGC 3885.

\section{Discussion and conclusions}

According to the ionized-gas velocity field, we distinguish three classes of objects in sample galaxies:

1. In NGC 4984, NGC 7213, and NGC 7377 an overall gas velocity curve is observed along the minor axis without zerovelocity points, out to the last measured radius. These gas kinematics have been interpreted as due to the warped inner structure of the gaseous disk.
2. In NGC 3885, NGC 4224, and NGC 4586 the gas velocity rises almost linearly in the inner regions of both the major (where gas rotates faster than stars) and minor axes. Nonzero gas velocities along the minor axis are confined in the nuclear regions. Such gas kinematics have been explained as being due to non-circular motion induced by a triaxial potential.

3. In NGC 2855 and NGC 7049 the major-axis gas velocity gradient in the innermost $\approx 500 \mathrm{pc}$ (where gas rotates slower than stars) is shallower than that measured farther out (where gas rotates faster than stars). Moreover the nonzero gas velocities observed along the minor axis are confined in the nuclear regions. These kinematic features have been attributed to the presence of an inner polar disk of gas.

Each of the above categories is discussed in detail in the following sections.

\subsection{Warped gaseous disks}

An inner warp of the gaseous disk can account for the gas velocity curve observed along the minor axes in the low-inclined galaxies NGC 4984, NGC 7213, and NGC 7377.

NGC 4984 is classified as a very early-type Sa spiral in CAG and as a weakly-barred S0/a in RC3. Sandage \& Bedke (1994) pointed out that this galaxy has a peculiar structure characterized by two sets of spiral arms. The inner spiral pattern is made by multiple dust arms (see the inset of Panel 73 in CAG) and shows a strong variation of the isophotal position angle from $\approx 20^{\circ}$ to $\approx 90^{\circ}$ in the innermost $30^{\prime \prime}$. This feature results 
from the near-infrared photometry by Jungwiert et al. (1997) who interpreted it as the possible signature of a double bar. A second set of spiral arms is visible outside the main body of the galaxy both in Panel 73 of the CAG and in the DSS plate reprinted in Fig. 1. The position angle of the outermost arms is $\approx 25^{\circ}$. This change of position angle is associated with a variation of the inclination from $i \approx 73^{\circ}$ at $\approx 80^{\prime \prime}$ to $i \approx 56^{\circ}$ at $\approx 130^{\prime \prime}$, and it is indicative of a warped disk. Therefore NGC 4984 has a peculiar structure with a double bar in the central $30^{\prime \prime}$ and a warped disk at larger radii. To perform our spectroscopic observations we adopted for the position angle of the major axis PA $=90^{\circ}$. This value is given by RC3. Our kinematic data extend out to $\approx 40^{\prime \prime}$ from the galaxy center on both the observed axes $\left(\mathrm{PA}=0^{\circ}, 90^{\circ}\right)$, which do not correspond to the actual photometric major and minor axis of the galaxy in the measured radial range.

A geometrical decoupling between the gaseous and stellar disks characterizes NGC 7213 in the radial region we covered with our spectroscopic observations $\left(\$ 40^{\prime \prime}\right)$. Indeed, the orientation of the circumnuclear ring-like structure observed in the map of the $\mathrm{H} \alpha$ emission obtained by Hameed \& Devereux (1999) does not coincide with that of the stellar disk as derived from the near-infrared photometry by Mulchaey et al. (1997). The ionized-gas ring has a diameter of $\approx 21^{\prime \prime}$, a major-axis position angle $\mathrm{PA} \approx 40^{\circ}$ and an inclination $i \approx 30^{\circ}$. According to Mulchaey et al. (1997) the isophotal ellipticity is about zero out to $\approx 40^{\prime \prime}$, suggesting that in this region the stellar disk is almost face-on with a major-axis position angle of $\approx 80^{\circ}$. This is consistent with our kinematic data, since we measure small rotation velocities for the stellar component $\left(V_{\star} \lesssim 50 \mathrm{~km} \mathrm{~s}^{-1}\right)$ along both the observed axes $\left(\mathrm{PA}=34^{\circ}, 124^{\circ}\right)$. These axes can be actually considered as intermediate axes. At larger radii the inclination and the major-axis position angle of the disk are $i=27^{\circ}$ and PA $=124^{\circ}$, respectively (see Table 1 ). A giant filament of ionized gas is located at $\approx 20 \mathrm{kpc}$ south of the galaxy (Hameed \& Devereux 1999) and the HI velocity field reveals NGC 7213 to be a highly disturbed system undergoing an acquisition event (Hameed et al. 2001). Therefore we conclude that the kinematic and photometric properties of NGC 7213 are consistent with a warped gaseous disk in the context of an ongoing merge process.

According to Sandage \& Bedke (1994) NGC 7377 is one of the most unusual galaxies in their atlas. They state that there is no evidence of recent star formation over an otherwise normal S0 disk, yet the entire disk is threaded by a multi-armed spiral pattern composed of dust lanes only. These lanes are more visible to the south-west (Panel 75, CAG). The morphology and the kinematics of NGC 7377 (Fig. 2) are similar to those of NGC 7213. In particular the major and minor-axis gas kinematics of NGC 7377 behave very similarly to the minor and major-axis gas kinematics of NGC 7213, respectively. So again a warp of the gaseous disk can be suspected. We interpret the counterrotation of gas and stars observed along the galaxy major axis and the gas velocity decrease along the minor axis (which is steeper than Keplerian) as due to a geometrical decoupling between the gaseous and stellar disks, which are not coplanar.

\subsection{Non-circular gas motions in a triaxial bulge or in a bar}

Non-zero gas velocities are measured along the minor axis in the bulge-dominated region of NGC 3885, NGC 4224, and NGC 4586, and a velocity gradient is observed along their major axis too. We interpret the observed kinematics as due to non-circular gas motion in the principal plane of the disk caused by bulge triaxiality or due to the action of a bar. Indeed the intrinsic shape of bulges is generally triaxial and the gaseous disk lies on the principal plane perpendicular to the bulge short axis (Bertola et al. 1991). Gas settled onto a principal plane of a non-rotating triaxial potential moves onto closed elliptical orbits. These orbits become nearly circular as soon as the distance from the center increases (de Zeeuw \& Franx 1989; Gerhard et al. 1989). The same is true in barred potentials too (Athanassoula 1992). The central velocity gradients measured along both major and minor axis of NGC 3885 , NGC 4224, and NGC 4586 are due to the orientation of the inner elliptical orbits lying on the disk plane and seen at intermediate angle between their intrinsic major and minor axes. Minor-axis gas rotation velocity drops to zero where elliptical orbits become circular.

The triaxiality of the bulge of NGC 3885 is suggested by the significant Lindblad's misalignment (Lindblad 1956) between the apparent major axes of bulge and disk $\left(\triangle \mathrm{PA} \approx 10^{\circ}\right)$. The bulge-to-disk misalignment measured on the photographic plates of NGC 3885 available in CAG (Panel 76 and 87) is consistent with the isophotal twist found in the near infrared by Jungwiert et al. (1997) in spite of the presence of the dust lanes threading through the smooth disk and crossing the bulge close to its center. Therefore the twist between the isophotes of bulge and disk of NGC 3885 cannot be attributed to the bulge obscuration by dust.

Both NGC 4224 and NGC 4586 have a boxy/peanut-shape bulge (Panel 76, CAG). These bulges can be interpreted as bars seen nearly edge on (Kuijken \& Merrifield 1995; Bureau \& Athanassoula 1999). This supports the idea that the gas velocity gradient observed along their minor axis is due to the noncircular gas motion in a triaxial potential. This is in agreement with the recent results by Athanassoula \& Bureau (1999), who studied by means of hydrodynamical simulations the appearance of the position-velocity diagram of the gaseous component in edge-on barred galaxies as a function of the viewing angle.

The presence of a gas velocity gradient along both the major and minor axis allows us to disentangle the case of gas noncircular motion from that of an inner gaseous disk in orthogonal rotation with respect to the galaxy disk. Indeed, as discussed in Sect. 4.3, the velocity field of a disk galaxy hosting in its center an orthogonally-rotating gaseous component is characterized by the presence of both a velocity gradient along the galaxy minor axis and a zero-velocity plateau along its major axis. To illustrate this point, in Fig. 3 we show the ionized-gas rotation curves along the galaxy major and minor axis in a triaxial bulge viewed from various directions. We considered the gas motion in the family of non-rotating triaxial mass models with a central density cusp studied by de Zeeuw \& Carollo (1996). They are 


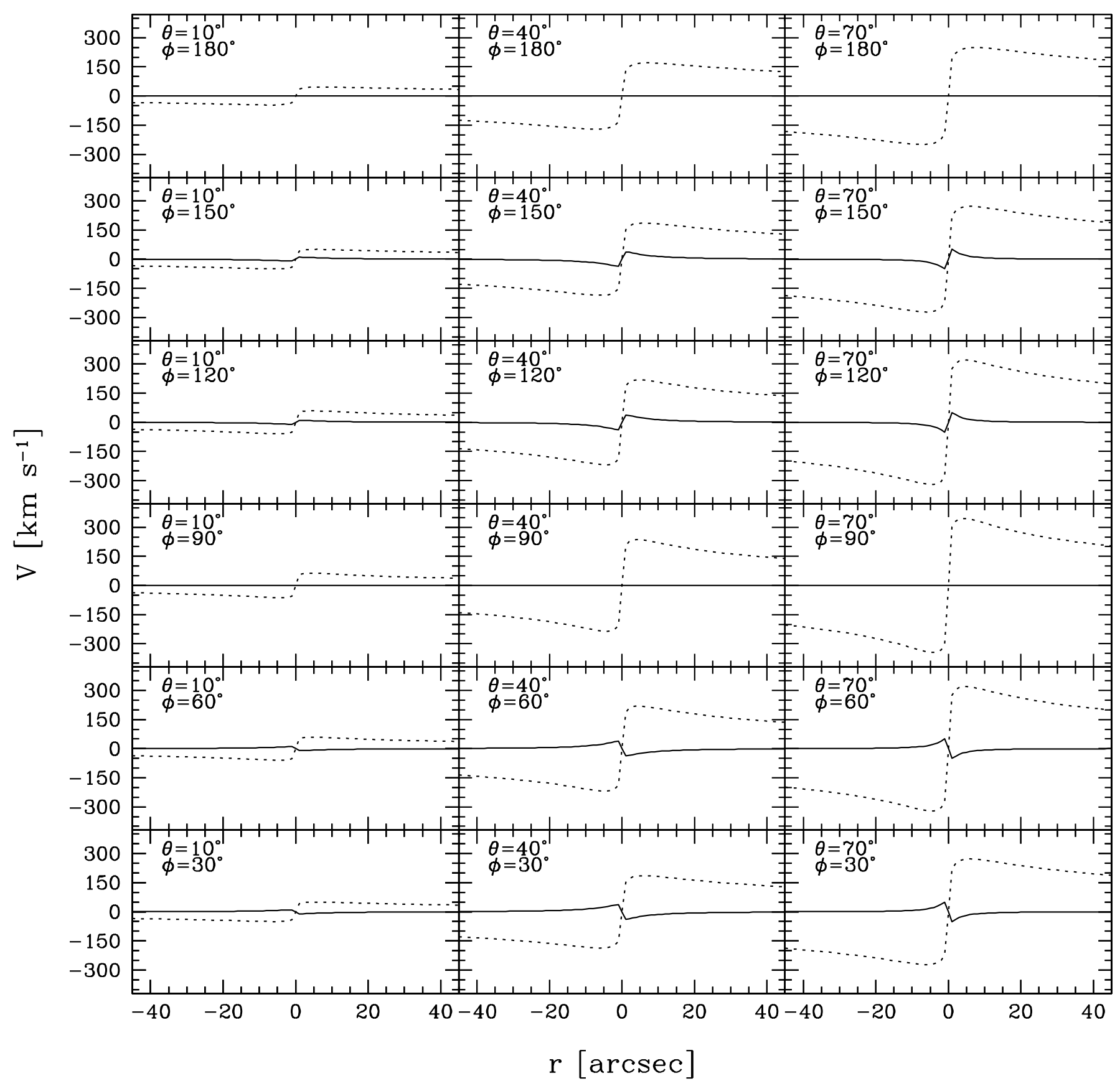

Fig. 3. Ionized-gas kinematics along the galaxy major (dotted line) and minor (continuous line) axis moving onto the plane perpendicular to the short axis of a triaxial bulge with axial ratios $p=0.7$ and $q=0.6$, total mass $M=7 \times 10^{10} M_{\odot}$, scalelength $r_{\mathrm{e}}=1.5 \mathrm{kpc}$, and density profile cuspiness $\gamma=1.5$ at a distance $D=25 \mathrm{Mpc}$. The viewing angles $\theta$ and $\phi$ are specified in each panel.

a generalization of the spherical $\gamma$-models with density profile

$\rho(r)=\frac{(3-\gamma)}{4 \pi} \frac{M r_{0}}{r^{\gamma}\left(r+r_{0}\right)^{4-\gamma}}$,

(see Dehnen 1993 and references therein), where $\gamma$ is the cuspiness of the central density profile $(0 \leq \gamma<3), M$ is the total mass of the galaxy, and $r_{0}$ is a scalelength which is related to the galaxy effective radius $r_{\mathrm{e}}$. The spherical models are made triaxial by adding two low-order spherical harmonic terms to the potential. The intrinsic shape of the mass density depends on $\left(p_{0}, q_{0}\right)$ and $\left(p_{\infty}, q_{\infty}\right)$, which are the axial ratios of the triaxial surfaces of constant density at small and at large radii, respectively. In the absence of figure rotation, settled gas is rotating either onto the plane perpendicular to the short axis (if $1>p>q>0$ ), or onto the plane perpendicular to the long axis (if $q>1>p>0$ ). For large ranges of flattenings and cusp slopes, the gas velocity field in the above potentials are accurately described by a first-order epicyclic treatment (de Zeeuw \& Carollo 1996). This approach has been used by Pizzella et al. (1997) to model the observed velocity field of the ionized-gas disks of a number of elliptical galaxies. 
We took into account a triaxial bulge with the short axis perpendicular to the stellar disk. We assumed the axial ratios to be the same at small and large radii $\left(p \equiv p_{0}=p_{\infty}\right.$, $q \equiv q_{0}=q_{\infty}$ ) and we adopted $p=0.7$ and $q=0.6$, which are the values observed for the Sa NGC 4845 (Bertola et al. 1986; Gerhard et al. 1986). We assumed $M=7 \times 10^{10} M_{\odot}$ and $r_{\mathrm{e}}=1.5 \mathrm{kpc}$ for the bulge total mass and effective radius, respectively. These are typical values for early-type bulges as derived from dynamical models based on stellar dynamics (Fillmore et al. 1986; Cinzano et al. 1999; Corsini et al. 1999; Pignatelli et al. 2001) and from surface photometry assuming a de Vaucouleurs profile (Kent 1985), respectively. For the model we assumed $D=25 \mathrm{Mpc}$, which is the mean distance of the sample galaxies in which the minor-axis rotation of the gas is confined to the bulge-dominated region. This distance corresponds to a scale of $121 \mathrm{pcarcsec}^{-1}$. Finally, we fixed $\gamma=1.5$ to approximate the bulge surface-brightness profile with a de Vaucouleurs law. We derived the gas velocity field at different viewing angles $(\theta, \phi)$ which are defined as the standard spherical coordinates of the line of sight in the bulge coordinate system. For each set of $(\theta, \phi)$ we extracted the gas rotation curve along the apparent major and minor axis of the galaxy disk (Fig. 3). It is evident from this figure that the noncircular gas motions induced by the bulge triaxiality give rise to non-zero gas velocities observed in the innermost regions of the galaxy minor axis. The amplitude and radial extension of this phenomenon are comparable to those we measured in NGC 3885, NGC 4224, and NGC 4586 if an intermediate inclination is considered. None of the simulated gas velocity fields shows either a zero-velocity plateau or a change in the slope of the velocity gradient along the galaxy major axis similar to those observed in NGC 2855 and NGC 7049.

\subsection{The case of inner polar gaseous disks}

The gas velocity field of NGC 2855 and NGC 7049 differs from that of NGC 3885, NGC 4224, and NGC 4586 since the velocity gradient observed along the minor axis is associated with a change in the slope of the velocity gradient measured along the major axis (which is shallower in the center and steeper away from the nucleus). This is the kinematic signature of the presence of two decoupled gaseous components rotating around two roughly orthogonal axes. Taking into account the triaxiality of bulges, these two orthogonally-decoupled components have to be settled onto the two principal planes of the host bulge. The outer gaseous component lies on the plane perpendicular to the short axis of the bulge, which is the one containing the galaxy disk. The inner gaseous component rotates in the plane perpendicular to the long axis of the bulge, and therefore it is in orthogonal rotation with respect to the galaxy disk. For this reason we observe in the bulge-dominated region a zero-velocity plateau along the disk major axis (or at least a shallower velocity gradient depending on the amount of decoupled gas and spatial resolution of the kinematic data) and a velocity gradient along the disk minor axis. The innermost gas component can be identified with an inner polar gaseous disk.
In both galaxies we exclude that the velocity gradient measured along the minor axis is due to non-circular gas motion in a barred potential. We do not observe any bar structure in the low-inclined disk of NGC 2855 neither on the optical image by Corsini et al. (2002) nor in the near-infrared images by Peletier et al. (1999) and Möllenhoff \& Heidt (2001). The same is true for NGC 7049 according to the color map by Veron-Cetty \& Veron (1988). In spite of looking more like to an elliptical than any of the other sample galaxies, NGC 7049 is a disk galaxy as it results from the photometric decomposition of the $r$-band surface photometry by Bertola et al. (1990, 1991).

Galaxies hosting an inner polar disk are a new class of objects, since these orthogonally-decoupled structures have been discovered in the last few years. The investigation of their structural properties and formation processes offers some clues about the processes driving secular evolution of gaseous and stellar components in galaxy centers. In order to get an exhaustive picture of the phenomenon, we compiled a list of disk galaxies with an inner polar disk from both data of the present paper and the literature. The main properties of both the host galaxies and their inner polar components are given in Table 5. For comparison we also included in Table 5 IC 1689 and UGC 5900, which are characterized by a small polar ring rather than an inner polar disk.

Inner polar disks are disks of small size $(R \approx 300 \mathrm{pc})$, which are located in the center of lenticular and spiral galaxies and are rotating in a plane perpendicular to that of the main disk of their host. Inner polar gaseous disks have been reported in all the galaxies listed in Table 5, except for NGC 4672 (Sarzi et al. 2002) where the orthogonally-rotating component is made up only of stars. Gas and stars are observed in the inner polar disks of NGC 2681 (Moiseev et al. 2003) and NGC 4698 (Bertola et al. 1999; Bertola \& Corsini 2000; Pizzella et al. 2002).

The radial extent of inner polar disks is small with respect to that of their host galaxy $\left(d / D_{25} \approx 0.03\right)$. They are smaller than the so-called "inner" polar rings found in IC 1689 $\left(d / D_{25}=0.24\right.$, Reshetnikov et al. 1995) and UGC 5900 $\left(d / D_{25}=0.14\right.$, Karataeva et al. 2001). On the other hand, the diameter of "classical" polar rings is comparable or even larger than that of the galaxies they surround $\left(d / D_{25} \approx 1-3\right.$, see Sackett 1991).

According to morphological classification and isophotal analysis of their host galaxies, the presence of an inner polar disk is not directly related either to the presence of a main bar or to that of a nuclear bar. NGC 2217, NGC 4845 and NGC 5850 are the only bona-fide barred spirals of the sample in Table 5. An intermediate-scale bar has been recognized in NGC 2841 by Afanasev \& Sil'chenko (1999). Nuclear bars have been photometrically detected in NGC 2217 (Jungwiert et al. 1997), NGC 2681 (Wozniak et al. 1995; Erwin \& Sparke 1999), NGC 3368 (Jungwiert et al. 1997), and NGC 5850 (Buta \& Crocker 1993; Wozniak et al. 1995), although only the nuclear bar of NGC 3368 has been kinematically confirmed by Moiseev et al. (2003) by means of integral-field spectroscopy.

The short dynamical time (few Myr) we derived for the galaxy regions where we observe the orthogonal rotation implies that inner polar disks are already settled in an equilibrium configuration. We exclude the possibility taken into 
Table 5. Galaxies with inner polar disks.

\begin{tabular}{|c|c|c|c|c|c|c|c|c|c|}
\hline \multirow{3}{*}{$\begin{array}{l}\text { Name } \\
\text { (1) }\end{array}$} & \multicolumn{2}{|c|}{ Morphological type } & \multirow{3}{*}{$\begin{array}{c}D \\
{[\mathrm{Mpc}]} \\
(4)\end{array}$} & \multirow{3}{*}{$\begin{array}{c}i \\
{\left[{ }^{\circ}\right]} \\
(5)\end{array}$} & \multirow{3}{*}{$\begin{array}{l}\text { Inner polar component } \\
\text { (6) }\end{array}$} & \multirow{3}{*}{$\begin{array}{c}R \\
{[\mathrm{pc}]} \\
(7)\end{array}$} & \multirow{3}{*}{$\begin{array}{c}d / D_{25} \\
(8)\end{array}$} & \multirow{3}{*}{$\begin{array}{c}\text { Nuclear bar } \\
\text { (9) }\end{array}$} & \multirow{3}{*}{$\begin{array}{l}\text { Ref. } \\
(10)\end{array}$} \\
\hline & [RSA] & [RC3] & & & & & & & \\
\hline & (2) & (3) & & & & & & & \\
\hline Arp 220 & & $\mathrm{~S} ?$ & 72.1 & 77 & gaseous disk & 100 & 0.01 & no & 1 \\
\hline NGC 253 & $\mathrm{Sc}(\mathrm{c})$ & $\mathrm{SABc}(\mathrm{s})$ & 3.4 & 79 & gaseous ring & 150 & 0.01 & & 2 \\
\hline NGC 2217 & $\mathrm{SBa}(\mathrm{s})$ & $(\mathrm{R}) \mathrm{SB} 0^{+}(\mathrm{rs})$ & 19.1 & 22 & gaseous disk & 190 & 0.01 & yes & 3,4 \\
\hline NGC 2681 & $\mathrm{Sa}$ & $\left(\mathrm{R}^{\prime}\right) \mathrm{SABa}(\mathrm{rs})$ & 13.3 & 25 & gaseous/stellar disk & 130 & 0.02 & $?$ & $5,6,7$ \\
\hline NGC 2841 & $\mathrm{Sb}$ & $\mathrm{Sb}(\mathrm{r})$ & 9.8 & 64 & gaseous disk & 190 & 0.02 & no & 8,9 \\
\hline NGC 2855 & $\mathrm{Sa}(\mathrm{r})$ & (R)S0/a(rs) & 22.3 & 27 & gaseous disk & 220 & 0.03 & no & 10,11 \\
\hline NGC 3368 & $\operatorname{Sab}(a)$ & $\mathrm{SABab}(\mathrm{rs})$ & 9.8 & 47 & gaseous disk & 100 & 0.01 & yes & 7,12 \\
\hline NGC 4548 & $\mathrm{SBb}(\mathrm{rs})$ & $\mathrm{SBb}(\mathrm{rs})$ & 17.0 & 37 & gaseous disk & 250 & 0.02 & no & 13 \\
\hline NGC 4672 & & $\mathrm{Sa}(\mathrm{s})$ pec sp & 39.9 & 75 & stellar disk & 580 & 0.05 & & 14,15 \\
\hline NGC 4698 & $\mathrm{Sa}$ & $\mathrm{Sab}(\mathrm{s})$ & 17.0 & 65 & gaseous/stellar disk & 250 & 0.03 & no & $15,16,17$ \\
\hline NGC 5850 & $\mathrm{SBb}(\mathrm{rs})$ & $\mathrm{SBb}(\mathrm{r})$ & 32.4 & 30 & gaseous disk & 630 & 0.03 & $?$ & $5,7,18$ \\
\hline NGC 6340 & $\mathrm{Sa}(\mathrm{r})$ & $\mathrm{S} 0 / \mathrm{a}(\mathrm{s})$ & 19.8 & 26 & gaseous disk & 480 & 0.05 & no & 19 \\
\hline NGC 7049 & $\mathrm{SO}_{3}(4) / \mathrm{Sa}$ & $\mathrm{SO}(\mathrm{s})$ & 29.6 & 47 & gaseous disk & 430 & 0.02 & no & 11 \\
\hline NGC 7217 & $\mathrm{Sb}(\mathrm{r})$ & $(\mathrm{R}) \operatorname{Sab}(\mathrm{r})$ & 16.4 & 35 & gaseous disk & 240 & 0.03 & no & 20,21 \\
\hline NGC 7280 & & $\mathrm{SAB}^{+}$ & 26.3 & 52 & gaseous disk & 260 & 0.03 & no & 22 \\
\hline IC 1689 & & $\mathrm{SO}(\mathrm{s})$ & 63.0 & 55 & gaseous/stellar ring & 2140 & 0.24 & & 23,24 \\
\hline UGC 5600 & & S0? & 37.6 & 46 & gaseous ring & 1090 & 0.14 & & 25,26 \\
\hline
\end{tabular}

Notes. - Column 2: morphological classification from RSA. Column 3: morphological classification from RC3. Column 4: distance either from the listed references or from Col. 20 in RSA assuming $H_{0}=75 \mathrm{~km} \mathrm{~s}^{-1} \mathrm{Mpc}^{-1}$. Column 5: inclination of the galaxy disk either from listed references or from the observed axial ratio $R_{25}$ (RC3) after correcting for intrinsic axial ratio as in Guthrie (1992). Column 6: properties of the inner polar component. Column 7: radius of the inner polar component corresponding to the maximum observed velocity. Column 8: ratio of the inner polar component to galaxy size. Galaxy isophotal diameters are from RC3. Column 9: presence of a nuclear bar. ? = uncertain. Column 10: references for kinematic and photometric data. 1 = Eckart \& Downes (2001); 2 = Anantharamaiah \& Goss (1996); $3=$ Bettoni et al. (1990); 4 = Jungwiert et al. (1997); $5=$ Wozniak et al. (1995); $6=$ Erwin \& Sparke (1999); $7=$ Moiseev et al. (2003); $8=$ Sil'chenko et al. (1997); 9 = Afanasev \& Sil'chenko (1999); 10 = Corsini et al. (2002); 11 = this paper; $12=$ Sil'chenko et al. (2003); $13=$ Sil'chenko (2002); 14 = Sarzi et al. (2000); 15 = Bertola \& Corsini (2000); 16 = Bertola et al. (1999); 17 = Pizzella et al. (2002); $18=$ Buta \& Croker (1993); 19 = Sil'chenko (2000); 20 = Zasov \& Sil'chenko (1997); 21 = Sil'chenko \& Afanasev (2000); 22 = Afanasev \& Sil'chenko (2000); 23 = Reshetnikov et al. (1995); 24 = Hagen-Thorn \& Reshetnikov (1997); 25 = Karataeva et al. (2001); 26 = Shalyapina et al. (2002).

account by Sil'chenko (2002) that gas of inner polar disks is moving on anomalous orbits in a triaxial bulge (or bar) that is tumbling about its short axis. In fact we do not observe gas in retrograde motion relative to stars at large radii from the galaxy center, where the gas orbits are expected to be highly inclined with respect to the figure rotation axis (see van Albada et al. 1982; Friedli \& Benz 1993 for details). We therefore suggest that inner polar disks lie in the principal plane perpendicular to the long axis of the triaxial bulge (or of the bar when it is present).

As for "classical" polar rings (see the recent numerical results by Bournaud \& Combes 2003), the acquisition of external gas via merging or accretion on nearly polar orbits by a pre-existing galaxy has been usually proposed to account for the formation of kinematically-decoupled components like inner polar disks (see list of references in Table 5). In some objects this scenario is supported by different arguments. The S0/a NGC 2681 underwent some kind of widely distributed starburst not earlier than $1 \mathrm{Gyr}$ ago, which has been ascribed to the dumping of tidally extruded gas from a galaxy neighbor (Cappellari et al. 2001). The Sa NGC 2855 is surrounded by a ring-like structure elongated in a direction close to the galaxy minor axis, which is indicative of a second event (Corsini et al. 2001). The same is true for the Sab NGC 3368 (Sil'chenko et al. 2003) which is close to the supergiant intergalactic ring of neutral hydrogen found in the Leo I group by Schneider et al. (1983). The large-scale distribution of the neutral hydrogen of the SBb NGC 5850 has been explained as due to an high-speed encounter with its companion NGC 5846 (Higdon et al. 1998). The Sab NGC 7217 hosts a counterrotating stellar disk, which is suggestive of a retrograde accretion from the environment (Kuijken \& Merrifield 1994).

Sil'chenko (2001) argued that the inner polar disks of NGC 2841, NGC 4548, NGC 6340 and NGC 7217 have an internal origin as their hosts are isolated spirals with a large amount of normally-rotating gas, and invoking the qualitative scenario proposed by Sofue \& Wakamatsu (1994) to put the gas on the polar orbits in the center of barred galaxies. However, all these arguments do not exclude an external origin of the decoupled component. Bettoni et al. (2001) proved that the environment of galaxies that experienced past gas accretion do not appear statistically different from those of normal galaxies. 
This means that second events are not ruled out by the isolation of the host galaxy. A large amount of accreted gas can sink toward the galaxy nucleus, sweeping away the pre-existing gas and giving origin to the decoupled component. This kind of interaction between newly acquired and pre-existing gas has been proposed to account for the reversal of rotation measured in the gaseous disk of NGC 4826 (Rubin 1994). Finally, the dynamical process investigated by Sofue \& Wakamatsu (1994) does not prevent the gas driven to the galaxy center from having been acquired.

To address the frequency of inner polar disks, a kinematic survey of a complete sample of nearby bulges is highly desirable. Indeed their small size prevents statistics based on morphological classification by visual inspection of photographic plates as done for classical polar rings (Whitmore et al. 1990). The measurement of the gas velocity field at high spatial resolution in the center of disk galaxies by state-of-the-art integralfield spectrographs (e.g. SAURON and MPFS, see the volume edited by Rosado et al. 2003 for a review) represents the ideal tool for this kind of investigation.

Acknowledgements. We are indebted to R. Bender and R. Saglia for providing us with the FCQ package we used for measuring the stellar kinematics. We thank A. Moiseev for helpful discussions, and O. Sil'chenko for making available her data in advance of publication. This research has made use of the Lyon-Meudon Extragalactic Database (LEDA) supplied by the LEDA team at CRAL-Observatoire de Lyon (France) and Digitized Sky Survey (DSS) produced at the Space Telescope Science Institute under U.S. Government grant NAG W-2166.

\section{References}

Afanasiev, V. L., \& Sil'chenko, O. K. 1999, AJ, 117, 1725

Afanasiev, V. L., \& Sil'chenko, O. K. 2000, AJ, 119, 126

Anantharamaiah, K. R., \& Goss, W. M. 1996, ApJ, 466, L13

Athanassoula, E. 1992, MNRAS, 259, 345

Athanassoula, E., \& Bureau, M. 1999, ApJ, 522, 699

Bender, R. 1990, A\&A, 229, 441

Bender, R., Saglia, R. P., \& Gerhard, O. E. 1994, MNRAS, 269, 785

Berman, S. 2001, A\&A, 371, 476

Bertola, F., \& Corsini, E. M. 1999, in Galaxy Interactions at Low and High Redshift, ed. J. Barnes, \& D. B. Sanders (Dordrecht: Kluwer Academic Press), IAU Symp., 186, 149

Bertola, F., \& Corsini, E. M. 2000, in From Early Universe to the Present, ed. F Combes, G. A. Mamon, \& V. Charmandaris (San Francisco: ASP), ASP Conf. Ser., 197, 115

Bertola, F., Rubin, V. C., \& Zeilinger, W. W. 1989, ApJ, 345, L29

Bertola, F., Vietri, M., \& Zeilinger, W. W. 1990, in Bulges of Galaxies, ed. B. J. Jarvis, \& D. M. Terndrup (Garching: ESO), 275

Bertola, F., Vietri, M., \& Zeilinger, W. W. 1991, ApJ, 374, L13

Bertola, F., Cinzano, P., Corsini, E. M., Rix, H., \& Zeilinger, W. W. 1995, ApJ, 448, L13

Bertola, F., Corsini, E. M., Vega Beltrán, J. C., et al. 1999, ApJ, 519, L127

Bettoni, D., Fasano, G., \& Galletta, G. 1990, AJ, 99, 1789

Bettoni, D., Galletta, G., \& Prada, F. 2001, A\&A, 374, 83

Binney, J. 1992, ARA\&A, 30, 51

Bournaud, F., \& Combes, F. 2003, A\&A, 401, 817

Bosma, A. 1991, in Warped Disks and Inclined Rings around Galaxies, ed. S. Casertano, P. Sackett, \& F. H. Briggs (Cambridge: CUP), 181
Briggs, F. H. 1990, ApJ, 352, 15

Buta, R., \& Crocker, D. A. 1993, AJ, 105, 1344

Bureau, M., \& Athanassoula, E. 1999, ApJ, 522, 686

Cappellari, M., Bertola, F., Burstein, D., et al. 2001, ApJ, 551, 197

Cinzano, P., Rix, H.-W., Sarzi, M., et al. 1999, MNRAS, 307, 433

Coccato, L., Corsini, E. M., Pizzella, A., \& Bertola, F. 2003, A\&A, in preparation

Corsini, E. M., Pizzella, A., Sarzi, M., et al. 1999, A\&A, 342, 671

Corsini, E. M., Pizzella, A., \& Bertola, F., 2002, A\&A, 382, 488

Dehnen, W. 1993, MNRAS, 265, 250

de Vaucouleurs, G., de Vaucouleurs, A., Corwin, H. G. Jr., et al. 1991, Third Reference Catalogue of Bright Galaxies (New York: Springer-Verlag) (RC3)

de Zeeuw, P. T., \& Carollo, C. M. 1996, MNRAS, 281, 1333

de Zeeuw, T., \& Franx, M. 1989, ApJ, 343, 617

Eckart, A., \& Downes, D. 2001, ApJ, 551, 730

Emsellem, E., \& Arsenault, R. 1997, A\&A, 318, L39

Erwin, P., \& Sparke, L. S. 1999, ApJ, 521, L37

Fillmore, J. A., Boroson, T. A., \& Dressler, A. 1986, ApJ, 302, 208

Freedman, W. L., Madore, B. F., Mould, J. R., et al. 1994, Nature, 371, 757

Friedli, D., \& Benz, W. 1993, A\&A, 268, 65

Galletta, G. 1996, in Barred Galaxies, ed. R. Buta, D. A. Crocker, \& B. G. Elmegreen (San Francisco: ASP), ASP Conf. Ser., 91, 429

Gerhard, O. E., \& Vietri, M. 1986, MNRAS, 223, 377

Gerhard, O. E., Vietri, M., \& Kent, S. M. 1989, ApJ, 345, L33

Gerhard, O., Jeske, G., Saglia, R. P., \& Bender, R. 1998, MNRAS, 295, 197

Guthrie, B. N. G. 1992, A\&AS, 93, 255

Hagen-Thorn, V. A., \& Reshetnikov, V. P. 1997, A\&A, 319, 430

Hameed, S., \& Devereux, N. 1999, AJ, 118, 730

Hameed, S., Blank, D. L., Young, L. M., \& Devereux, N. 2001, ApJ, 546, L97

Higdon, J. L., Buta, R. J., \& Purcell, G. B. 1998, AJ, 115, 80

Jungwiert, B., Combes, F., \& Axon, D. J. 1997, A\&AS, 125, 479

Karataeva, G. M., Yakovleva, V. A., Hagen-Thorn, V. A., \& Mikolaichuk, O. V. 2001, AstL, 27, 74

Kent, S. M. 1985, ApJS, 59, 115

Kormendy, J. 1982, ApJ, 257, 75

Kuijken, K., \& Merrifield, M. R. 1995, ApJ, 443, L13

Lindblad, B. 1956, Stockholms Observatoriums Annaler, 2

Merrifield, M. R., \& Kuijken, K. 1994, ApJ, 432, 575

Moiseev, A. V., Valdés, J. R., \& Chavushyan, V. H. 2003, A\&A, submitted

Möllenhoff, C., \& Heidt, J. 2001, A\&A, 368, 16

Mulchaey, J. S., Regan, M. W., \& Kundu, A. 1997, ApJS, 110, 299

Peletier, R. F., Knapen, J. H., Shlosman, I., et al. 1999, ApJS, 125, 363

Pignatelli, E., Corsini, E. M., Vega Beltrán, J. C., et al. 2001, MNRAS, 323,188

Pizzella, A., Amico, P., Bertola, F., et al. 1997, A\&A, 323, 349

Pizzella, A., Corsini, E. M., Morelli, L., et al. 2002, ApJ, 573, 131

Reshetnikov, V. P., Hagen-Thorn, V. A., \& Yakovleva, V. A. 1995, A\&A, 303, 398

Rosado, M., Binette, L., \& Arias, L. 2003, Galaxies: The Third Dimension (San Francisco: ASP), ASP Conf. Ser. 282

Rubin, V. C. 1994, AJ, 107, 173

Sackett, P. D. 1991, in Warped Disks and Inclined Rings around Galaxies, ed. S. Casertano, P. Sackett, \& F. Briggs (Cambridge: CUP), 73

Sandage, A., \& Bedke, J. 1994, The Carnegie Atlas of Galaxies (Washington: Carnegie Institution) (RSA)

Sandage, A., \& Tammann, G. A. 1981, A Revised Shapley-Ames Catalog of Bright Galaxies (Washington: Carnegie Institution) (CAG) 
Sarzi, M., Corsini, E. M., Pizzella, A., et al. 2000, A\&A, 360, 439 Schneider, S. E., Helou, G., Salpeter, E. E., \& Terzian, Y. 1983, ApJ, 273, L1

Shalyapina, L. V., Moiseev, A. V., \& Yakovleva, V. A. 2002, AstL, 28, 443

Sil'chenko, O. K. 2000, AJ, 120, 741

Sil'chenko, O. K. 2002, AstL, 28, 207

Sil'chenko, O. K. 2003, in Galaxies: The Third Dimension, ed. M. Rosado, L. Binette, \& L. Arias (San Francisco: ASP), ASP Conf. Ser., 282, 121

Sil'chenko, O. K., \& Afanasiev, V. L. 2000, A\&A, 364, 479

Sil'chenko, O. K., Vlasyuk, V. V., \& Burenkov, A. N. 1997, A\&A, 326, 941
Sil'chenko, O. K., Moiseev, A. V., Afanasiev, V. L., Chavushyan, V. H., \& Valés, J. R. 2003, ApJ, in press [astro-ph/0303432] Sofue, Y., \& Wakamatsu, K. 1994, AJ, 107, 1018

van Albada, T. S., Kotanyi, C. G., \& Schwarzschild, M. 1982, MNRAS, 198, 303

Veron-Cetty, M.-P., \& Veron, P. 1988, A\&A, 204, 28

Whitmore, B. C., Lucas, R. A., McElroy, D. B., et al. 1990, AJ, 100, 1489

Wozniak, H., Friedli, D., Martinet, L., Martin, P., \& Bratschi, P. 1995, A\&AS, 111, 115

Zasov, A. V., \& Sil'chenko, O. K. 1997, ARep, 41, 734 